\title{
Determination of magnetoelastic oscillations in ferrite plate with different values of anisotropy constant
}

\author{
Leonid Kotov ${ }^{1}$, Pavel Severin ${ }^{1, *}$, Vladimir Vlasov ${ }^{1}$ and Dmitry Beznosikov ${ }^{1}$ \\ ${ }^{1}$ Syktyvkar State University named after Pitirim Sorokoin, Komi Republic, Syktyvkar 167000, Russia
}

\begin{abstract}
The maximum amplitudes of magnetic and elastic oscillations are calculated at various material parameters and parameters of external fields. Normally and tangentially magnetized anisotropic magnetic plates were considered. To obtain the maximum amplitudes with additional constraints, the simulated annealing method was used. The behaviour of the magnetic and elastic components of the oscillations was considered. The changes in the position of the magnetization vector and the equilibrium displacements as a function of the first anisotropy constant were revealed.
\end{abstract}

\section{Introduction}

Analysis of parameters of differential equations with the account for the boundary conditions can be reduced to more general problem of finding the extrema of the cost function, which is a reflection of specific simulated characteristics (for example, the degree of randomness of the excited oscillations, the amplitude of the elastic and magnetic oscillations). For global optimization simulated annealing method is used to solve a large class of problems. The advantage of the method is that, even with limited computing resources, it allows the extremum to be found that is close to the global one [1].

The nonlinear magnetoelastic dynamics of films and plates is a rapidly developing region [2-6]. Thus, the use of optimization methods in the study of magnetoacoustic interactions is also an urgent task.

Since the experimental search for the maxima of the amplitudes of elastic oscillations is usually hampered by the lack of high-power radio frequency generators and sensitive receivers with low noise, then this calculation allows to narrow down the frequency and amplitude of the ranges of external magnetic fields, in which it is necessary to carry out the study. Thus the solution of this problem allows reducing financial and time expenses in nonlinear magnetoelastic tasks.

In this study, the simulated annealing method is used to find a set of parameters of a system of differential equations describing the excitation of oscillations by a radio-frequency magnetic field. For these parameters, the smallest change in the constants of the cubic magnetic anisotropy corresponds to the largest change in the amplitudes of the elastic oscillations. The regions with high sensitivity of the amplitude of the elastic oscillations to the changes of the anisotropy constants were observed.
Further we consider the behavior of the magnetic and elastic components at the founded points of the multidimensional parameter space of the model.

\section{Basic equations}

The complete system of differential equations for the problem of excitation of magnetoelastic oscillations of radio frequency magnetic field in the normally magnetized ferrite plate is written following [2].

The plane-parallel plate with a thickness $d$ having magnetic, elastic and magnetoelastic properties is considered. External DC magnetic field $\mathbf{H}_{\mathbf{0}}$ is perpendicular to the plane of the plate and the alternating magnetic field vector $\mathbf{h}_{\mathbf{0}}$ is tangential to the surface of the plate. The problem is solved in a Cartesian coordinate system Oxyz. The plane $O x y$ of the coordinate system coincides with the plane of the plate. The coordinate axes are parallel to the edges of the cube crystallographic cell. The center of the coordinate system $\mathrm{O}$ is in the center of the plate, so that the plate planes coordinates are $z= \pm d / 2$, as shown in Figure 1 .

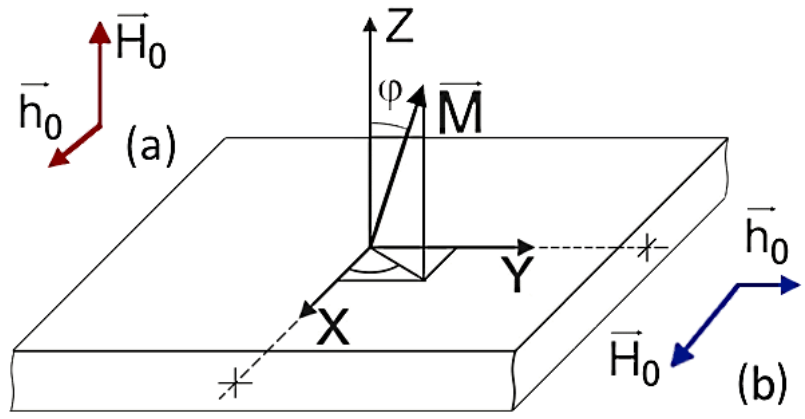

Fig. 1. Direction of magnetic fields and magnetic moment for a normally magnetized plate (a). Direction of magnetic fields (b) for a tangentially magnetized plate (the magnetic moments in this case are directed along the $O x$ axis) 
Assuming that the total energy density of the plate $U$ in the field $\mathbf{H}=\left(h_{x} ; h_{y} ; H_{0}\right)$ equals the sum of magnetic, elastic and magnetoelastic energy densities, we obtain [2]:

$$
\begin{aligned}
& U=-M_{0} h_{x} m_{x}-M_{0} h_{y} m_{y}-M_{0} H_{0} m_{z}+2 \pi M_{0}^{2} m_{z}^{2} \\
& +K_{1}\left(m_{x}^{2} m_{y}^{2}+m_{x}^{2} m_{z}^{2}+m_{z}^{2} m_{y}^{2}\right)+K_{2} m_{x}^{2} m_{y}^{2} m_{z}^{2} \\
& +2 c_{4}\left(u_{x y}^{2}+u_{y z}^{2}+u_{z x}^{2}\right)+ \\
& 2 B_{2}\left(m_{x} m_{y} u_{x y}+m_{y} m_{z} u_{y z}+m_{z} m_{x} u_{z x}\right),
\end{aligned}
$$

where, $\mathbf{m}=\mathbf{M} / M_{0}$ is the normalized magnetization vector, $M_{0}$ is the saturation magnetization, $K_{1}, K_{2}-$ the first and second magnetocrystalline anisotropy constants, $u_{i j}$ are components of the deformation tensor, $c_{44}$ is the constant of elasticity and $B_{2}$ is the constant of magnetoelastic interaction. The system of the ordinary differential equations describing the magnetoelastic oscillations are obtained in [2].

The system describes the excitation of the transverse elastic oscillations. The initial equations for the obtained system are the Landau-Lifshitz-Gilbert equation and the equations for the elastic displacement vector components $u_{x, y}$ :

$$
\begin{aligned}
& \frac{\partial \vec{m}}{\partial t}=-\gamma \cdot\left[\vec{m} \times \vec{H}_{e}\right]+\alpha \cdot\left[\vec{m} \times \frac{\partial \vec{m}}{\partial t}\right], \\
& \frac{\partial^{2} u_{x, y}}{\partial t^{2}}=-2 \beta \frac{\partial u_{x, y}}{\partial t}+\frac{c_{44}}{\rho} \frac{\partial^{2} u_{x, y}}{\partial z^{2}},
\end{aligned}
$$

where effective fields are:

$$
\begin{aligned}
& H_{e x}=h_{x}+\frac{B_{2}^{2}}{M_{0} c_{44}} m_{x} m_{z}^{2}-\frac{2 B_{2}}{M_{0} d} m_{z} v_{x}- \\
& \frac{2 K_{1}}{M_{0}} m_{x}\left(m_{y}^{2}+m_{z}^{2}\right)-\frac{2 K_{2}}{M_{0}} m_{x} m_{y}^{2} m_{z}^{2}, \\
& H_{e y}=h_{y}+\frac{B_{2}^{2}}{M_{0} c_{44}} m_{y} m_{z}^{2}-\frac{2 B_{2}}{M_{0} d} m_{z} v_{y}- \\
& \frac{2 K_{1}}{M_{0}} m_{y}\left(m_{x}^{2}+m_{z}^{2}\right)-\frac{2 K_{2}}{M_{0}} m_{y} m_{x}^{2} m_{z}^{2}, \\
& H_{e z}=H_{0}-4 \pi M_{0} m_{z}+\frac{B_{2}^{2}}{M_{0} c_{44}} m_{z}\left(m_{x}^{2}+m_{y}^{2}\right)- \\
& \frac{2 B_{2}}{M_{0} d} m_{x} v_{x}-\frac{2 B_{2}}{M_{0} d} m_{y} v_{y}- \\
& \frac{2 K_{1}}{M_{0}} m_{z}\left(m_{x}^{2}+m_{y}^{2}\right)-\frac{2 K_{2}}{M_{0}} m_{z} m_{x}^{2} m_{y}^{2} .
\end{aligned}
$$

The equations for the elastic displacement are [2]:

$$
u_{x, y}=-\frac{B_{2}}{c_{44}} m_{x, y} m_{z} z+v_{x, y} \sin \left(\frac{\pi}{d} z\right)
$$

Boundary conditions are:

$$
\left.c_{44} \frac{\partial u_{x, y}}{\partial z}\right|_{z= \pm d / 2}=-B_{2} m_{x, y} m_{z} .
$$

In case of a tangentially magnetized ferrite plate, the geometry of the problem is described as follows: a DC external magnetic field $\mathbf{H}_{\mathbf{0}}$ is directed along $O x$ axis and the alternating magnetic field $\mathbf{h}_{\mathbf{0}}$ is directed along $O y$ axis.

The system of the equations is solved numerically by 7th to 8th order Runge-Kutta-Fehlberg method with control of the length of the integration step.

Reliability of the results of modeling is provided by use of universally recognized methods and the correctness of the mathematical formulation of the problem $[1-2,7]$.

\section{Parameters of manganese-zinc spinel}

To investigate the processes with different values of anisotropy constants a manganese-zinc spinel (MZS)

\begin{tabular}{|c|c|c|c|c|c|c|}
\hline \multirow{2}{*}{$\begin{array}{c}T \\
(\mathbf{K})\end{array}$} & \multirow{2}{*}{$\begin{array}{l}M_{s} \\
(\mathbf{G})\end{array}$} & $K_{I} \times 10^{-3}$ & $K_{2} \times 10^{-3}$ & $B_{2} \times 10^{-6}$ & $C_{44} \times 10^{-11}$ & \multirow{2}{*}{$\alpha \times 10^{2}$} \\
\hline & & \multicolumn{4}{|c|}{$\left(\mathrm{erg} \cdot \mathrm{cm}^{-3}\right)$} & \\
\hline 450 & 18 & 0.35 & -1.09 & -3.43 & 10.88 & 14.8 \\
\hline 400 & 125 & 1.41 & -1.15 & -11.96 & 10.58 & 12.9 \\
\hline 350 & 225 & 2.82 & -1.15 & -20.42 & 10.45 & 11.3 \\
\hline 300 & 318 & 3.32 & -2.88 & -30.21 & 10.27 & 10.1 \\
\hline 263 & 383 & -0.03 & -6.26 & -39.65 & 9.99 & 9.4 \\
\hline 250 & 404 & -2.94 & -7.80 & -44.56 & 10.01 & 9.2 \\
\hline 200 & 482 & -22.21 & -15.07 & -64.42 & 9.63 & 8.3 \\
\hline 150 & 550 & -64.77 & -19.30 & -90.30 & 9.72 & 7.5 \\
\hline 100 & 608 & -133.90 & -24.21 & -115.71 & 9.78 & 6.7 \\
\hline 50 & 653 & -229.59 & -34.00 & -140.97 & 9.80 & 6.0 \\
\hline 30 & 666 & -275.31 & -39.80 & -150.96 & 9.80 & 5.8 \\
\hline 10 & 675 & -325.28 & -46.27 & -160.96 & 9.80 & 5.5 \\
\hline
\end{tabular}
crystal grown by the Verneuil method with a specific composition $\mathrm{Mn}_{0.61} \mathrm{Zn}_{0.35} \mathrm{Fe}_{2.04} \mathrm{O}_{4}$ was chosen.

The choice is due to the availability of experimental works [7-8], as well as available characteristics for this and similar in composition crystals [7].

Dependences of the anisotropy constants and other parameters of MZS on temperature are taken from [7], as well as from experimental data (the velocity of a shear elastic wave) and are presented in Table 1. We used a linear extrapolation based on the available data to obtain the values of the parameters in the low-temperature region. The Curie temperature for the selected material is $T_{c}=458 \mathrm{~K}$.

Table 1. Parameters of MZS in dependence on temperature.

The thickness of the plate and the density of the plate material was chosen as: $\rho=5.4 \mathrm{~g} \mathrm{~cm}^{-3}, d=7.69 \times 10^{-5} \mathrm{~cm}$. The oscillations were excited by an alternating magnetic field pulses. The pulse duration was 10 periods of excitation frequency and the amplitude was $0.01 \mathrm{Oe}$. 
The evolution of the oscillations was considered in a the time interval 10 times larger than pulse duration. The damping parameter of the elastic system is typical for MZS crystal: $\beta=1.5 \times 10^{6} \mathrm{~s}^{-1}$.

In order to reduce the oscillation development time to a stationary amplitude and to exclude short-term relaxation effects from the simulation, the damping parameter of the magnetic system was taken as $\alpha=0.5$.

\section{Using simulated annealing}

We take $\mathrm{P}=\left(M_{s}, f, \Delta H\right)$ as the set of parameters of a system of differential equations describing the excitation of oscillations by a radio-frequency magnetic field, at which the smallest change in the constants of the crystallographic anisotropy $K_{1}$ and $K_{2}$ would correspond to the largest change in the amplitudes of the elastic vibrations $u_{x}$.

We denote $\Delta K_{1}$ and $\Delta K_{2}$ as the desired changes in the anisotropy constants, and $\triangle K_{1}^{M A X}$ and $\triangle K_{2}^{M A X}$ as the maximum possible changes determined by the boundaries of the search area. The cost function was used in simulated annealing algorithm. The maximum of the function should be found by formula:

$$
\begin{gathered}
F=\left|u_{x}\left(P, K_{1}, K_{2}\right)-u_{x}\left(P, K_{1}+\Delta K_{1}, K_{2}+\Delta K_{2}\right)\right| \\
\left(1-\sqrt{\frac{\Delta K_{1}^{2}+\Delta K_{2}^{2}}{\Delta K_{1}^{2 M A X}+\Delta K_{2}^{2 M A X}}}\right) \\
\left(1-\frac{\left|K_{1}\right|+\left|K_{1}+\Delta K_{1}\right|}{\left|2 \Delta K_{1}^{M A X}\right|}\right)\left(1-\frac{\left|K_{2}\right|+\left|K_{2}+\Delta K_{2}\right|}{\left|2 \Delta K_{2}^{M A X}\right|}\right)
\end{gathered}
$$

The cost function implicitly contains the maximization condition for $\mathrm{u}_{\mathrm{x}}$. The remaining factors describe the additional conditions of the extremum and are normalized. It is necessary to ensure the equilibrium of these conditions in the search for the maximum of the cost function.

The ranges of parameters changes are: frequency of alternating magnetic field $f$ varied from $300 \mathrm{MHz}$ to $3000 \mathrm{MHz}$; the DC field exceeded the saturation magnetization field $4 \pi M_{s}$ by an amount $\Delta H$ from 100 Oe to $1000 \mathrm{Oe}$. The anisotropy constants and the saturation magnetization varied within the limits corresponding to a change in temperature from 10 to $450 \mathrm{~K}$. We change these parameters to search the maximum of the elastic displacements.

The remaining parameters were taken from Table 1 for a temperature $T=263 \mathrm{~K}\left(K_{l} \approx 0\right)$.

The values of the input parameters which correspond to two states of the model was found using the simulation annealing (Figure 2).

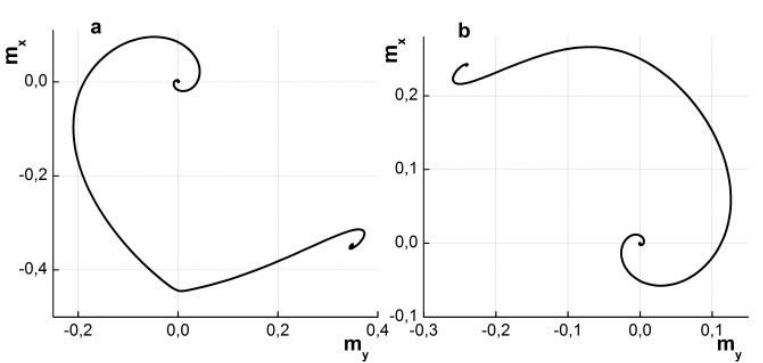

Fig. 2. Precession patterns for the case of a normally magnetized plate with $f=1359 \mathrm{MHz} ; M_{s}=163.05 \mathrm{G}$, $\Delta H=971.83 \mathrm{Oe}$ and $u_{x}=5.12 \times 10^{-10} \mathrm{~cm}, K_{l}=-181398 \mathrm{erg} \mathrm{cm}^{-3}$, $K_{2}=-1090 \mathrm{erg} \mathrm{cm}^{-3}(\mathrm{a}) ; \quad u_{x}=6.96 \times 10^{-12} \mathrm{~cm}, \quad K_{I}=-114143$

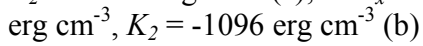

\section{Numerical solution}

Let us consider the behavior of the elastic component and the dependence of the precession angle $\varphi$ on $K_{l}$ (In this geometry, changes in $K_{2}$ lead to changes in $u_{x}$ in order of $10 \%$ ).

It is seen from Figure 3 that the change in the anisotropy constant $K_{1}$ of about $40 \%$ entails a change in the final position of the magnetization vector during reorientation (Figure 2) and also a significant change the amplitude of the elastic oscillations.

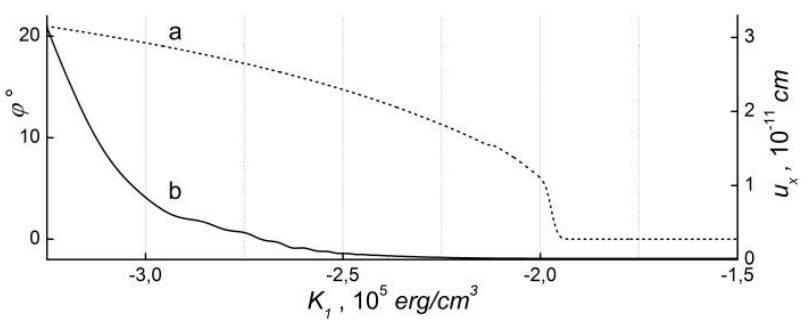

Fig. 3. Dependence of $\varphi$ (a) and $u_{x}$ (b) on $K_{l}$ for the case of a normally magnetized plate

The mean-square values of the oscillation amplitude were calculated without taking into account the constant component in the considered time interval (which included the region of excitation of the oscillations).

The threshold value $K_{1}$ for these values of $M_{s}$ and $\Delta H$ is about $-1.8 \times 10^{5} \mathrm{erg} \mathrm{cm}^{-3}$. The reorientation occurs at $K_{I}$ smaller than this value. The increase of the amplitude of the elastic oscillations is observed.

Figure 4 shows that the DC field $H_{0}$ and the saturation magnetization $M_{s}$ influence to the threshold value $K_{l}$. 

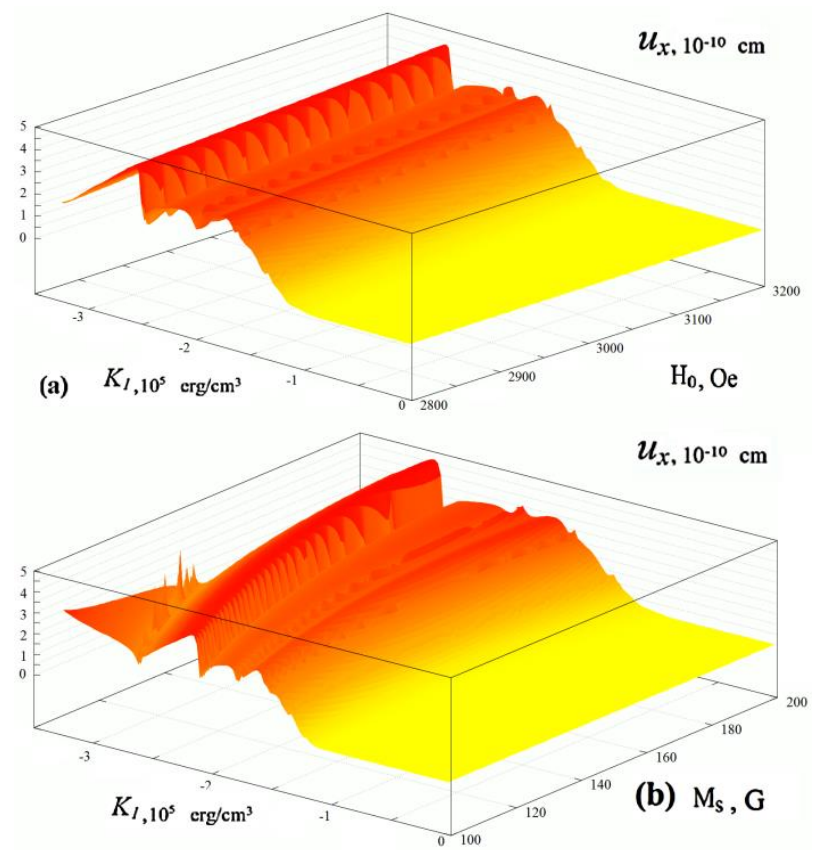

Fig. 4. Dependence of $u_{x}$ amplitude on $K_{l}$ and $H_{0}$ (a) and on $K_{I}$ and $M_{S}$ (b) for the case of a normally magnetized plate

Thus, the threshold character of the change in the amplitude of the elastic oscillations can be explained by the interaction of the anisotropy field with the magnetization field.

The calculation of the average value of the amplitude of the magnetic component (Figure 5) makes possible to estimate its final position at the end of the reorientation.
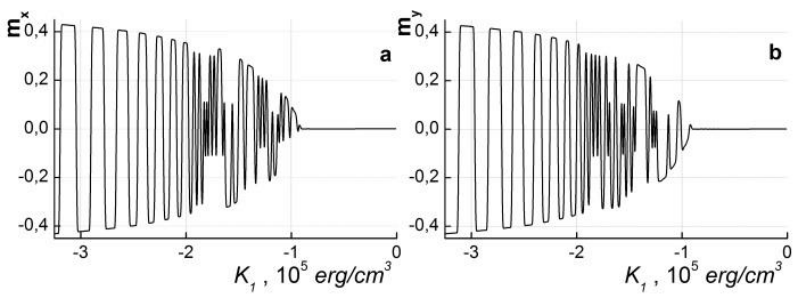

Fig. 5. Average value of the amplitude of the magnetic components $m_{x}$ (a) and $m_{y}$ (b) on $K_{l}$ for the case of a normally magnetized plate, $\alpha=0.05$

It is seen that as the module $K_{I}$ increases, the final position of magnetization deviates from zero, while spontaneous changes in the direction of the projection of the magnetization vector onto the corresponding axis are observed. Changes in the direction are especially frequent for values of $K_{l}$ close to the threshold.

It should be noted that for the geometry of a normally magnetized plate considered above, the precession angle is $0^{\circ}-20^{\circ}$.

Let us consider the case of tangentially magnetized plate. We use pulse excitation with short pulses duration $5.2 \times 10^{-9} \mathrm{~s}$ and amplitude 1 Oe to avoid parametric decay processes. Parameters that differ from those used in the geometry of a normally magnetized plate are: the observation time $10^{-7} \mathrm{~s}, \quad d=1.098 \times 10^{-4} \mathrm{~cm}$, $f=1958 \mathrm{MHz}, H_{0}=100 \mathrm{Oe}$.

The dependence of the mean-square amplitudes of the elastic oscillations and the precession angle $\varphi$ on $K_{l}$ is shown in Figure 6.

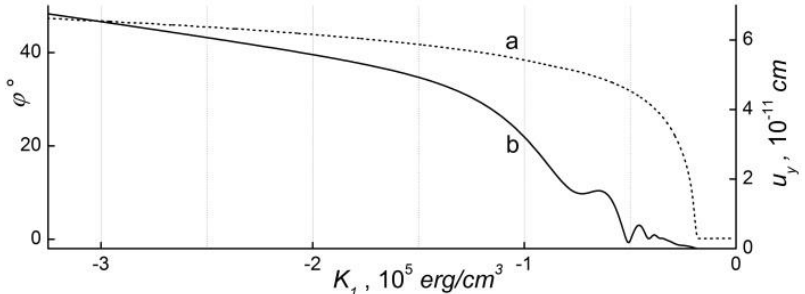

Fig. 6. Dependence of $\varphi$ (a) and $u_{y}$ (b) on $K_{l}$ for the case of a tangentially magnetized plate

The amplitude of the elastic oscillations is slightly larger than the amplitude in the geometry of the normally magnetized plate. But the amplitude of the alternating field 100 times larger than in the geometry of the normally magnetized plate.

Thus the efficiency of elastic oscillations excitation is far better in the geometry of the normally magnetized plate.

\section{Conclusions}

The behavior of the amplitudes of magnetic and elastic oscillations was described in the manganese-zinc spinel monocrystalline plate. The optimization method simulating annealing was used. The system of magnetoelastic equations was solved using 7 th to 8 th order Runge-Kutta-Fehlberg method. Precession patterns and other characteristics for the components of magnetization and elastic displacements were obtained as a function of the anisotropy constants. The value of the first anisotropy constant $-1.8 \times 10^{5} \mathrm{erg} \cdot \mathrm{cm}^{-3}$ was found at which a reorientation of the magnetization vector occurs under the action of a small amplitude alternating field. The changes in the position of the magnetization vector and the equilibrium displacements as the function of the first anisotropy constant were revealed.

The support from RFBR (grants \#\# 17-02-01138, 17-57$150001)$ is gratefully acknowledged.

\section{References}

1. L. Ingber, Control Cybern. 25, 33 (1996)

2. V.S. Vlasov, L.N. Kotov, V.G. Shavrov, V.I. Shheglov, J. Communic. Technol. Electron. 54, 863 (2009)

3. Nicolas Tiercelin et.al. Proc. SPIE, Spintronics X, 103571T (2017).

4. Kim J.W., Bigot J.Y. Phys. Rev. B 95, 144422 (2017).

5. Kim J. W., Vomir M., Bigot J. Y. Scientific reports 5, 8511 (2015).

6. L. Thevenard et al. Phys. Rev. B 87, 144402 (2013).

7. B.A. Goldin, L.N. Kotov, L.K. Zarembo, S.N. Karpachov, Spin-phonon interactions in crystals (1991)

8. L. N. Kotov, S. N. Karpachev, Tech. Phys. Lett. 28, 105 (2002). 